\title{
VM Migration Techniques in Cloud: A Review
}

\author{
Taruna khemwani \\ Assistant Professor \\ Govt Women Engg College, Ajmer
}

\author{
Rakesh Rathi, PhD \\ Assistant Professor \\ Govt Engineering College, Ajmer
}

\begin{abstract}
Cloud computing is recent technology. In cloud environment services are provided in an ubiquitous, easy and on request fashion to share computing resources [4][5]. The key point in cloud computing is Virtualization[6][14], which provides the partition of one physical machine into multiple virtual machines that can run simultaneously. Physical resources and hardware are shared in virtualization [15] where applications are encapsulated within virtual machines (VM) is dynamically mapped onto a pool of physical servers. Live migration of virtual machines is a process of moving a virtual machine in its running state or application in it's running state between different physical machines, while not disturbing the service provided to the user. All the system resources, which are assigned to the virtual machine like memory, storage, process and Network resources like connectivity, are transferred from the original host machine to the destination machine. Load Balancing, Energy efficiency and fault tolerance which is also known as High availability of physical servers, in Cloud Data center are significant performance needs of Live Migration. This paper incorporates a detailed review on Migration of Virtual machines in cloud environment
\end{abstract}

\section{Keywords}

Cloud Computing, VM Migration, pre copy, post copy

\section{INTRODUCTION}

Cloud computing is latest technology in which Platform, software and Infrastructure are accessible as a service. The client getting to the service needs to pay just for the proportion he has utilized. Cloud computing works on virtualization technology and utility computing. Virtualization is a technology that provide an environment to enable numerous segregated and secure virtualized servers to run on a single physical server. With the use of virtualization a no. of virtual machines can be hosted on the same physical server for optimal utilization of resources, therefore subsequently diminishing the expense of deploying a Datacenter. It also enhances the security of physical servers in Data center. A virtual machine is a computer software that has its own operating system and application running on it like any other physical machine. The virtual machine has its own resources to provide various services. VM migration is a process in which the VM is moved from one physical machine (PM) to another physical machine (PM) [12]. VM migration originates from process Migration [8],[9], Efficient energy utilization [12][13], load balancing, fault Tolerance[8][11] and high availability of physical server in Cloud Data center can be counted as the main objectives for VM migration process.

However, this operation must be consistent to the users and should have no affect on the service experience of users [2].Total migration time, downtime, overall network traffic and service degradation are some of the performance measures for VM migration technologies [17]. As in recent scenario a large no. of clients select cloud data centers to execute the client applications [7], The efficient management of the VMs in a data center is a key point to discuss. This paper presents a review on the virtual machines migration patterns in cloud environment. It also discusses about the challenges in Live Virtual machine migration techniques.

Section II presents various categories of virtual machine migration techniques in Cloud environment according to the needs of virtual machine migration. Section III describes various live migration techniques cloud Data center. Section IV describes advantages and challenges with VM migration. Section V gives a conclusion of this research article.

\section{DIFFERENT CATEGORIES OF MIGRATION TECHNIQUES [3]}

Technique that migrate the entire OS and its associated application from one physical machine to another, is called Live Virtual machine Migration. In live migration, migration is to be done in the running state of virtual machine with no interruption on the executing application. The key merits of virtual machine migration incorporate power conservation of physical server, fault tolerance in case of sudden failure and the balancing of load in between the physical servers. Following section elaborate about the various kind of techniques for migration of a virtual machine

\subsection{Load Balancing Migration Techniques}

For the distribution of load across the physical servers in a manner that enhances the scalability of physical servers in cloud environment ,Load balancing migration techniques is used. This technique also provides implementation of fail-over, minimizes the resource consumption, avoid bottlenecks, enhances scalability, and also avoid over-provision of resources etc.

\subsection{Energy Efficient Migration Techniques}

In Data center the power is required for the utilization of the servers and their cooling systems. Most of the maximum power consumption of servers is consumed, even if these are less utilized level. Therefore techniques are needed to migrate for the conservation of power of servers and to optimize the utilization of resources.

\subsection{Fault Tolerant Migration Techniques}

In case of failure of any server part, Fault tolerance techniques provides solutions for the virtual machines to continue its job. In this technique, if there is a prediction of the failure occurrences then the virtual machine is migrated from existing physical server to another physical server. Thus fault tolerant migration technique is used to enhance the availability of physical server and diminish down turn in applications performance.

\section{VIRTUAL MACHINE MIGRATION}

To segregate the data center and server workload maintenance $\mathrm{VM}$ migration is required. It migrate the processor state (I/O, CPU, memory) between physical machines [1]. VM migration technique is used to balance the load among servers on the basis of available resources.VM migration is a good option when number of client increases in cloud data center, to provide a better service [3]. Basically for migration of VM two migration patterns are implemented such as live migration and non-live migration. In non-live migration, service provided is stopped in the duration of 
migration. In live migration, service provided remains unaffected in the duration of migration.

\subsection{Non-Live Migration}

Non live migration is easiest and newest migration technique. In this technique the current execution of VMs are suspended and resumed before and after the migration phases, respectively [1][2][17]. It is a simple technique as the service provided is stopped while migration process continues. the state of VM does not resume on the destination server until the migration process completes .Memory pages need to be migrated only once using this technique. The migration time is also very short and predictable. Although this technique simplifies the migration process but as the service provided is paused during the migration phase, application performance is degraded

\subsection{Live VM migration}

Live migration technique is the most commonly used for VM migration. Unlike to non-live VM migration, there is no interruption in service in live migration. Efficient utilization of bandwidth, To optimize the application performance, minimize the application downtime and reducing the overall time required for transfer by maximum factor, are the key objectives of live VM migration. For live migration of VM process and its memory states, there are two main approaches. These approaches with their working style and migration strategy are described in following section.

\subsubsection{Pre-Copy Migration[17]}

In Pre-copy migration, while keeping the VM in running condition ,the transfer of memory contents to the destination is done simultaneously. For pre-copy VM migration process there are mainly six steps, including: (1) Selecting the destination host,(2) Reservation of Resources, (3) Iterative pre-copying rounds, (4) Stop and copy phase, (5) Commitment, and (6) VM activation at the destination server.

The transfer phase continues if the memory content are changed in this duration, then these changed contents or dirty pages are continuously transferred to the destination. This process remains continue until one of the following two conditions are met
1) Fixed threshold is reached
2) Remaining no. of pages is small.

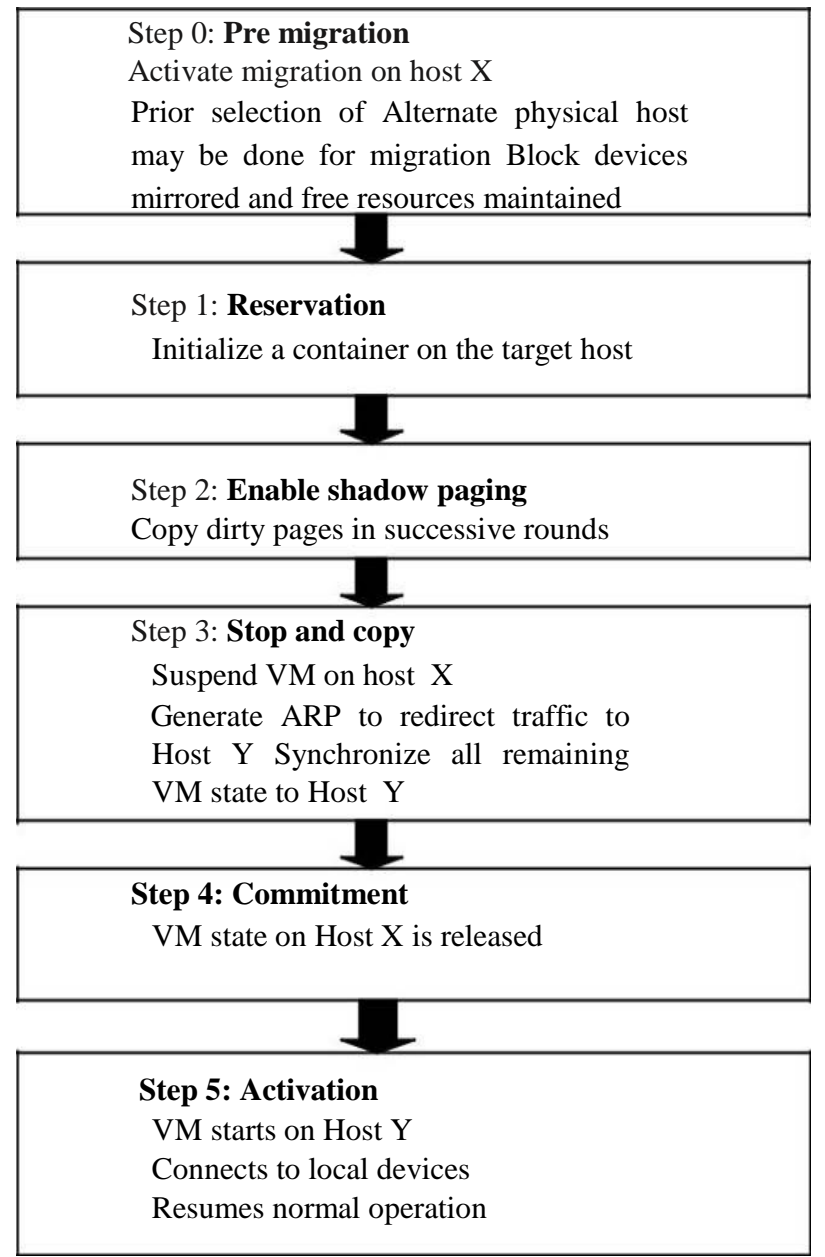

Fig:1 Pre copy migration process steps [3]

Now the VM is suspended and the remaining pages are copied

After that firstly the Execution of VM is started in the target machine, and the main VM is then terminated. Low VM downtime (required for copying the remaining dirty pages), which is main advantage of pre copy migration. On the other hand, repeated copying of dirty pages can increase the whole migration time . Pre copy migration is advantageous in cases of few memory transfers, but in case of more memory transfers greater transfer time and downtime will be observed.

\subsubsection{Post-Copy Migration[17]}

In Post-copy migration the memory contents are fetched after the transfer of process state to the destination server. The prior transfer of process states to the destination machine in this scheme enables the VM to resume instantly at the target machine. The memory contents of VM are copied to destination from source while VM is running on the target host. Memory faults are generated for the memory pages that have not been fetched yet, and now the missing pages are to be fetched from source machine. As excess and repetitive memory faults results in significant disruption in the service quality, so to reduce this type of service interruption techniques such as memory prepaging, are usually taken into account to lower the number of page faults. 


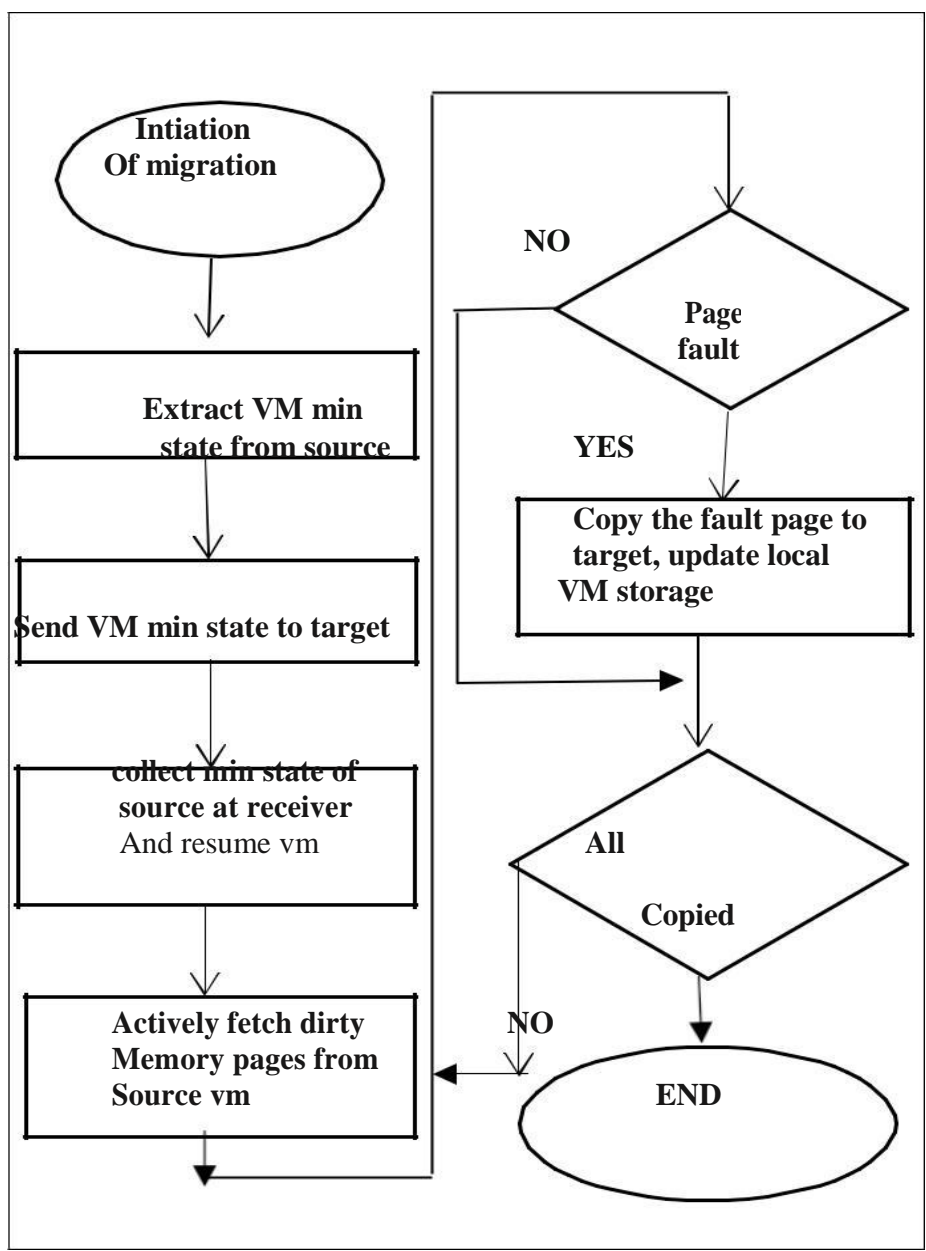

Fig 2: Post -copy migration flowchart.[18]

Memory prepaging provides high accuracy in predicting the subsequent memory uses by assuming that VM memory access reveals specific and time bounded location. Therefore, Memory faults can be significantly reduced by prior migration of the corresponding memory pages which has high demand probability,. Finally, Now the source VM does not have the latest memory contents, a failure during the migration process can likely result in irrecoverable VM states. Checkpoint mechanism is One of the best possible mechanism to remove this limitation that is to checkpoint the VM state from the destination VM back to the source VM. Overall, to lower the migration time is the key merit of post-copy migration, as in the whole process the memory contents are copied at most once. On the other hand, due to the occurrence of memory faults more service interruption can be there.

Attempts can be made to merge pre-copy and post-copy migration techniques into a hybrid migration technique. Notably, the precopy migration supports the prior transfer of memory contents to the destination. On completion, now the state of Virtual Machine is suspended, the dirty pages are now to be transferred to the destination machine. The VM execution is then resumed, with the new dirty pages pushed to the destination machine as in the postcopy migration. Larger no. of memory pages are copied in pre copy because of repeatedly copying memory pages for a certain number of iterations[3]. This combination can yield a better tradeoff between the merits and demerits of both.

\section{ADVANTAGES AND CHALLENGES OF VM MIGRATION}

4.1 Advantages The merits of VM migration can be put forth in following aspects

- Load balancing Migration is a good option in cases of some server overload under loaded VMs can be migrated in between for load balancing.

- Zero-downtime hardware maintenance VM migration works in scenario of server failure or suspension for hardware maintenance, the VMs can be migrated for this duration on another server and can be moved back after recovery[4][11].

- Server consolidation In cases of Energy or communication purpose VMs are migrated live[10] .

- Across-site management for the cloud providers managing more than one sites, improving Quality of service

- Hybrid cloud Public cloud can be loaded in peak load hours.

- Cloud federation For minimizing deficiencies cloud providers can cooperate with each other.

- Breaking vendor lock-in VM migration is good technique to avoid the user locked with one vendor using

- User's mobility reaction for uninterrupted service to user while he is moving from one place to another, related VM can be migrated on appropriate target.

\subsection{Challenges in VM migration}

- Network connection continuity. As a VM migrated to a different machine, it is required to be approachable to clients. For live migration, the open connections should also be remained alive during migration

- Memory data migration: All running states are required to be copied to the destination If after migration it is required for the migrated VM to run from the point where it was stopped. Memory data, states of device, states of CPU etc are included in data of state.

- Storage data migration. The transfer of virtual disk of migrated VM, on the target will be required in case if the storage system of the source data center is not reachable from the target server of VM migration. As high disk I/O latency can be there on remote access of disk data and same may also create violation of SLA.

\section{CONCLUSION}

This Paper presents a review on virtual machine migration techniques. The main categories of migration techniques are efficiency of power, balance of load and fault tolerance techniques. Non live and live migration are patters of migration. Non live migration reduces the migration time while interrupting the services provided. On thev other side live migration reduces the downtime and provide uninterrupted services to the user. Precopy and Post copy are the two variants of live migration pattern. Precopy provide proactive migration of required pages while post copy migrates VM first and then the pages are migrated as page faults occur. Pre copy migration is advantageous in cases of few memory transfer Advantages of VM migration like zero downtime, load balancing, sever consolidation, vendor lock in, users mobility are also discussed. At last major challenges with VM migration in case of Memory migration, storage data migration and network connection continuity are discussed. 


\section{REFERENCES}

[1] R. W. Ahmad, A. Gani, S H. Ab. Hamid, M. Shiraz, A.Yousafzai, and F. Xia, "A survey on virtual machine migration and server consolidation frameworks for cloud data centers," Journal of Network and ComputerApplications, 2015, vol. 52, pp. 11-25.

[2] V. Medina and J. Manuel Garcia, "A Survey of Migration Mechanisms of Virtual Machines," ACM ComputingSurveys, January, 2014, Vol. 46, No. 3, Article 30 , pp. 1-33.

[3] Mohammad A. Altahat*, Anjali Agarwal*, NishithGoel and Marzia Zaman "Analysis and Comparison of Live Virtual Machine Migration Methods" 6thInternational Conference on Future Internet of Things and Cloud 2018 (IEEE),PP 251258.

[4] T. Mather, S. Kumaraswamy, and S. Latif, Cloud security and privacy: an enterprise perspective on risks and compliance. " O'Reilly Media, Inc.”, 2009

[5] G. Boss, P. Malladi, D. Quan, L. Legregni, and H. Hall,"Cloud computing," IBM white paper, vol. 321, pp. 224-231, 2007.

[6] A.Weiss, "Computing in the clouds," Computing, vol. $16,2007$.

[7] L. Cheng, I. Tachmazidis, S. Kotoulas, and G. Antoniou, "Design and evaluation of small-large outer joins in cloud computing envi-ronments," Journal of Parallel and Distributed Computing, 2017.

[8] J. Wu, S. Guo, J. Li, and D. Zeng, "Big data meet green challenges: Greening big data," IEEE Systems Journal, vol. 10, no. 3, pp. 873-887, 2016.

[9] S. Osman, D. Subhraveti, G. Su, and J. Nieh, "The design and imple-mentation of zap: A system for computing environment,"ACM SIGOPS Operating Systems Review, Vol. 36, no. SI, pp. 361-376, 2002
[10] J. G. Hansen and A. K. Henriksen, "Nomadic operating systems," Ph.D. dissertation, Citeseer, 2002.

[11] P. Padala, X. Zhu, Z. Wang, S. Singhal, K. G. Shin et al., "Performance evaluation of virtualization technologies for server consolidation,” HP Labs Tec. Report, 2007.

[12] L Cheng and T. Li, "Efficient data redistribution to speedup big data analytics in large systems," in High Performance Computing (HiPC), 2016 IEEE 23rd International Conference on. IEEE, 2016, pp. 91- 100.

[13] R. Bianchini and R. Rajamony, "Power and energy management for server systems," Computer, vol. 37, no. 11, pp. 68-76, 2004.

[14] Amin Julaa, Alankovan, Sundararajanb,Zalinda Othmana. "Cloud computing service composition: systematic literature review", Expert Systems With Applications, (2014),Vol. 41, pp.3809-3824.

[15] A.Singh, M. Korupolu, and D. Mohapatra, "Server storage virtualization: integration and load balancing in data centers," in Proc. of the ACM/IEEE conference on Supercomputing, 2008, pp.1-53.

[16] Manel Bourguiba et al," Improving Network I/O Virtualization for Cloud Computing", IEEE Transactions On Parallel And Distributed Systems, March 2014, Vol. 25, No. 3, pp.673-681

[17] Fei Zhang, Guangming Liu, Xiaoming $\mathrm{Fu}$, Ramin Yahyapour "A survey on virtual machine migration: challenges, techniques and open issues" IEEE Communication survey and tutorial, vol 20 pp1206-1243.

[18] NAYAK, P.C. GARG,D. SHAKVA,A.KU. \&SAINI(2018) A Research paper of existing live migration A research paper of existing Live VM Migration and a Hybrid VM Migration approach in Cloud Computing 2018 2nd international conference on trends in electronics and informatics(ICOEI) 